Kurimasa et al.

\title{
Disruption of NBS1 gene leads to early embryonic lethality in homozygous null mice and induces specific cancer in heterozygous mice.
}

Akihiro Kurimasa ${ }^{14)}$, Sandeep Burma ${ }^{1)}$, Melinda Henrie ${ }^{1)}$, Honghai Ouyang ${ }^{2}$, Mitsuhiko Osaki $^{6}$, Hisao Ito ${ }^{6)}$, Hatsumi Nagasawa ${ }^{3)}$, John B. Little ${ }^{3)}$, Mitsuo Oshimura ${ }^{5}$, Gloria C. $\mathrm{Li}^{2)}$, and David J. Chen ${ }^{1) *}$

1) Life Sciences Division, Lawrence Berkeley National Laboratory, Berkeley, CA 94720

2) Department of Radiation Oncology, Department of Medical Physics, Memorial SloanKettering Cancer Center, New York, NY 10021

3) Department of Cancer Cell Biology, Harvard School of Public Health, Boston, MA

4) Department of Human Genome Sciences, Life Sciences Division, Graduate school of Medicine, Tottori University, Yonago, Tottori, JAPAN 683-8503

5) Department of Molecular and Cell Genetics, School of Life Sciences, Faculty of Medicine, Tottori University, Yonago, Tottori, JAPAN 683-8503

6) Department of Pathology(1), Faculty of Medicine, Tottori University, Yonago, Tottori, JAPAN 683-8503

Running Title: Embryonic lethality and cancer in NBS1 mice

*Corresponding Author:

David J. Chen, Ph.D.

Life Sciences Division

Lawrence Berkeley National Laboratory

1 Cyclotron Rd. MS74-157

Berkeley, CA 94720

Phone: 510-495-2861

Lab phone: $510-486-5861$

Fax: 510-486-6816

e-mail: djchen $(\alpha)$, lbl.gov

This work was supported by the U.S. Department of Energy under Contract No. DE-AC03-76SF00098. 
Kurimasa et al.

\begin{abstract}
Nijmegen breakage syndrome (NBS) is a rare autosomal recessive chromosomeinstability syndrome characterized by microcephaly, growth retardation, immunodeficiency, and cancer predisposition, with cellular features similar to that of ataxia telangiectasia (AT). NBS results from mutations in the mammalian gene $N b s 1$ that codes for a 95-kDa protein called nibrin, NBS1, or $\mathrm{p} 95$. To establish an animal model for NBS, we attempted to generate NBS1 knockout mice. However, NBS1 gene knockouts were lethal at an early embryonic stage. NBS1 homozygous(-/-) blastocyst cells cultured in vitro showed retarded growth and subsequently underwent growth arrest within 5 days of culture. Apoptosis, assayed by TUNEL staining, was observed in NBS1 homozygous(-/-) blastocyst cells cultured for four days. NBS1 heterozygous $(+/-)$ mice were normal, and exhibited no specific phenotype for at least one year. However, fibroblast cells from NBS1 heterozygous $(+/-)$ mice displayed an enhanced frequency of spontaneous transformation to anchorage-independent growth as compared to NBS1 wild-type $(+/+)$ cells. Furthermore, heterozygous(+/-) mice exhibited a high incidence of hepatocellular carcinoma after one year compared to wild-type mice, even though no significant differences in the incidence of other tumors such as lung adenocarcinoma and lymphoma were observed. Taken together, these results strongly suggest that NBS1 heterozygosity and reduced NBS1 expression induces formation of specific tumors in mice.
\end{abstract}


Kurimasa et al.

\section{INTRODUCTION}

Nijmegen breakage syndrome (NBS) is a rare autosomal recessive chromosomeinstability syndrome characterized by microcephaly, growth retardation, immunodeficiency, and cancer predisposition $(5,9,32)$. NBS was originally believed to be a variant form of ataxia telangiectasia (AT) because the characteristic features of NBS are very similar to that of AT. However, some of the characteristic features of AT such as ataxia, cerebellar degeneration and ocular telangiectasia are not seen in NBS patients, while the characteristic bird-like face accompanied by microcephaly are specific to NBS(5). Cancer incidence observed in NBS patients is higher than in AT, the risk for malignancy in NBS patients being as high as $34 \%(9)$. The prominent cancers observed in NBS patients are lymphoreticular malignancies such as $B$ and $T$ cell lymphomas, rhabdomyosarcoma, haemoblastoma, and neuroblastoma(32).

Cells from NBS patients show hypersensitivity to ionizing radiation (IR), chromosome instability, and radioresistant DNA synthesis $(\operatorname{RDS})(9,27,32)$. These cellular features are similar to that of AT. Complementation analysis can distinguish one cell type from another, while AT and NBS can be distinguished by clinical criteria. This suggests that the corresponding gene products function in the same pathway in response to DNA double-strand breaks (DSBs) which are typically generated by ionizing radiation(13).

The gene mutated in NBS was cloned by three different groups employing positional cloning within a critical region of chromosome $8 \mathrm{q} 21(23,34)$ or by identification of the gene encoding a 95-kDa protein that was part of the Mre11-Rad50 DSB repair complex(6). This novel mammalian gene $N b s 1$ codes for a $95-\mathrm{kDa}$ protein 
called nibrin, NBS1, or p95. NBS1 is part of the Mre11/Rad50 complex that associates with DNA DSBs in irradiated cells early in the cellular DNA-damage response, indicating that it may function in the early stages of detection and signaling of DNA damage. Due to the similarity of AT and NBS cellular phenotypes, it has been hypothesized that NBS and ATM act in a common DNA-damage response pathway. Indeed, ATM kinase has been shown to phosphorylate NBS1 in response to IR-induced DNA damage. This phosphorylation event is required for activation of an S-phase checkpoint $(12,18,37,40)$.

The Mre11/Rad50/NBS1 complex is believed to be a sensor of abnormal DNA structures and/or a regulator of the postreplication repair process. This complex is part of a large multi-subunit protein complex called BASC, for BRCA1-associated genome surveillance complex, consisting of various repair proteins including ATM, BLM, MSH2, MSH6, MLH1, and RFC1-RFC2-RFC4 complex(26, 35). Furthermore, the Mre11/Rad50/NBS1 complex is associated with telomeric repeat-binding factor TRF2, suggesting additional functions at the telomere, possibly involving modulation of t-loop formation(42). Mre11 and Rad50 form discrete nuclear foci in cells treated with DSBinducing agents. NBS1 deficiency in cells from NBS patients completely abrogates the formation of Mre11/Rad50 IR-induced foci(6). The abrogation of Mre11/Rad50/NBS1 protein complex in NBS cells indicates an important role for NBS1 in linking DSB repair and cell cycle checkpoint functions(26).

In order to establish an animal model for NBS, we performed gene targeting experiments to generate NBS1-null knockout mice. However, NBS1 gene knockouts were embryonic lethal at an early stage. Here we report the embryonic lethality of 
Kurimasa et al.

homozygous NBS1-null knockout embryos and characterize the potential oncogenic phenotype of NBS1 heterozygous mice. 
Kurimasa et al.

\section{MATERIALS AND METHODS}

\section{Construction of the targeting vector}

A genomic clone of NBS1 locus was isolated from 129/Sv mouse genomic library using two EST clones (IMAGE consortium clone ID: 567467 and 974211) of mouse NBS1 gene. Following restriction mapping and determination of intron-exon boundaries, restriction fragments were subcloned into pBluescriptIISK $(+)$ (Stratagene). Targeting vector was constructed using a PstI-AgeI 5.5kb fragment containing exon 2 to 5 and a PstI-NarI $1.2 \mathrm{~kb}$ promoter fragment, which were inserted into $\mathrm{pBluescriptIISK}(+)$ vector flanking either side of a PGK-neo cassette.

\section{Targeting of ES cells and generation of germ-line chimeras}

Transfection of the linealized targeting vector to CJ7 ES cells was performed by electroporation, and colonies surviving G418 $(200 \mu \mathrm{g} / \mathrm{ml})$ selection were screened by PCR and Southern blotting of EcoRI-digested genomic DNA. A 1.5kb PstI fragment 5' upstream of the promoter sequence was used as a probe. Correctly targeted ES clones were then injected into C57BL/6 blastocysts followed by transfer to a pseudopregnant foster mother to generate chimeric mice. The chimeras were crossed with C57BL/6 females, and germ-line transmission was demonstrated by the presence of agouti coat color in the F1 offspring. The presence of a disrupted NBS1 allele was confirmed by Southern blot analysis. NBS1 heterozygous mice were maintained by intercrossing. This study was conducted in compliance with all relevant federal guidelines and institutional policies on murine research. 
Kurimasa et al.

\section{Genotyping of NBS1 knockout mice}

The genotype of individual mice was determined by PCR, which distinguishes between the endogenous and targeted NBS1 alleles. PCR was performed in a $20 \mu \mathrm{l}$ reaction with 100ng of genomic DNA, $1 \mathrm{x}$ PCR buffer (Perkin-Elmer, $1.5 \mathrm{mM} \mathrm{MgCl} 2$ ), 0.5 $\mu \mathrm{M}$ (each) of primers: NB-27: 5'-TTC ATT GAT TAA AGC GTC CAG TT-3', NB-32: 5'-CAC TCA GGA TAC AGT GGA AAA GAT-3', NB-35: 5'-GAC GTG CGC GTT CCC AGG ACT CTG-3', Pol-8: 5'-GCA GCC TCT GTT CCA CAT ACA CTT-3', $0.2 \mathrm{mM}$ (each) of dNTPs, and 0.8U of AmpliTaq DNA polymerase. Primers NB-32 and NB-35 give a product of wild-type allele that is 335bp; primers NB-27 and Pol-8 yield a product of the targeted allele that is $161 \mathrm{bp}$.

The genotype of blastocysts and early stage embryos was determined by nested PCR. The PCR conditions used are the same as described above. Initial PCR was performed using mixed primers, NB-28: 5'-TTA AGG GAA ATT GGG ACT ACT TG3', NB-36: 5'-GAA AGA ACT CCC CGC CGC CTC CTT-3', Pol-7: 5'-TGA GGG GAT CCG CTG TAA GTC TGC-3'. The secondary primers for the wild-type allele are NB-32 and NB-35, which generate a 335 bp product as mentioned above and primers for the targeted allele are NB-32 and Pol-8, which gives a 331 bp product. After separation of PCR products by electrophoresis, amplified DNA was transferred to Hybond N+ membrane and visualized by hybridization with ${ }^{32} \mathrm{P}$-end labeled primer probes which specifically detect the wild-type (NB-34: 5'-ACT CTG ACA AGT TCT TCG GAA AGG-3' ) or the targeted allele (NB-27). 
Kurimasa et al.

\section{Establishment of primary ear fibroblasts}

Primary ear fibroblast cells were isolated from 4-week-old NBS1 wild-type $(+/+)$ and heterozygous $(+/-)$ sibling mice. Cells were maintained at $37^{\circ} \mathrm{C}$ in alpha-MEM medium supplemented with $10 \%$ fetal calf serum, $100 \mathrm{U} / \mathrm{ml}$ penicillin and $100 \mu \mathrm{g} / \mathrm{ml}$ streptomycin in a humidified atmosphere with $5 \% \mathrm{CO}_{2}$.

\section{Blastocyst culture and TUNEL assay}

e3.5 blastocysts were collected from NBS1 heterozygous(+/-) intercrosses, and rinsed in Dulbecco's modified $\mathrm{Mg} / \mathrm{Ca}$ free phosphate buffered saline (Gibco BRL). Blastocysts were cultured in 4-well chamber slides in $1 \mathrm{ml}$ of Dulbecco's modified Eagles medium (Gibco BRL) supplemented with 2mM L-glutamine, 1x non-essential amino acids, 1,000U/ml leukemia inhibitory factor (LIF), $100 \mathrm{U} / \mathrm{ml}$ penicillin, $100 \mu \mathrm{g} / \mathrm{ml}$ streptomycin, $0.1 \mathrm{mM}$ beta mercaptoethanol, and $20 \%$ fetal calf serum. Blastocysts were fixed in $4 \%$ paraformaldehyde after 1-3 days in culture. Permealization and TUNEL assay were performed using the Apoalert DNA fragmentation assay (Clontech) according to manufacturer's instructions with the following modification: a hydrophobic ring was drawn around the blastocyst and the assay was performed using micro-volumes of the sample. Samples were also stained with DAPI or PI. Images were taken at $200 \mathrm{x}$ and 400x magnification and captured using the Spot Diagnostic Imaging System (model:1.0.0) (Diagnostic Instrument Inc.) with Olympus BX60 microscope. 
Kurimasa et al.

\section{Colony formation assay in soft agar}

The ability of primary ear fibroblasts to grow in soft agar under anchorageindependent conditions was determined by use of a modified MacPherson method(21). Plastic petri dishes $(60 \mathrm{~mm})$ were coated with a layer of $5 \mathrm{ml}$ of $0.5 \%$ agarose (Sigma Type I) in regular medium. Two $\mathrm{ml}$ of the cell suspension was mixed with $4 \mathrm{ml}$ of the $0.5 \%$ agarose solution, and $1.5 \mathrm{ml}$ of the resulting cell suspension was spread over the basal agarose layer. Subsequently, the cultures were fed once a week by adding $1 \mathrm{ml}$ of complete medium (without agarose). Size of the colonies in soft agar was determined at 2 days and 1 month after seeding by taking photomicrographs of the cultures on an inverted microscope. The circumferences of 100 random colonies for each experimental point were traced, the diameters measured, and the volume determined for each soft agar colony.

\section{Histological analysis of NBS1 embryos and tumors derived from heterozygous(+/-) mice}

Whole embryos were observed and photographed under a dissecting microscope or a low power microscope. Embryonic stage was estimated using timed mating. Whole embryo sacs taken at e6.5 were fixed using $4 \%$ paraformaldehyde, sectioned for histopathological analysis, and stained by hematoxylin and eosin method.

For the tumor histopathological analysis from aging mice, tissues exhibiting pathological changes when inspected visually at autopsy were frozen at first in liquid nitrogen and then fixed in $10 \%$ formalin solution. Sectioned specimens were stained by hematoxylin and eosin method and analyzed for pathological diagnosis. 
Kurimasa et al.

\section{RESULTS}

\section{Generation of NBS1 gene knockout mouse}

The targeting vector for NBS1 gene was constructed by substituting the NBS1 promoter region (about 200bp upstream of ATG start codon), entire exon1, and $2 \mathrm{~kb}$ of intron1 with a PGK-neo cassette (Fig.1A). This substitution removed the transcription and translation initiation sites resulting in null expression of mouse NBS1 gene.

The linearized targeting vector was transfected into CJ7 ES cells. A single targeted ES allele was observed in 5 out of 400 isolated G418 resistant ES cell colonies (Fig.1B, C). Three targeted ES clones were injected into C57BL/6 blastocysts to generate chimeric mice. Only one of the three ES clones showed good contribution towards chimera formation as assayed by analysis of coat color. These chimeras were crossed with $\mathrm{C} 57 \mathrm{BL} / 6$ females to generate $\mathrm{F} 1$ progenitors. Among the $17 \mathrm{~F} 1$ mice that were obtained, 7 males and 5 females showed successful transmission of the targeted allele as assayed by PCR and Southern blot analysis. The F2 and F3 NBS heterozygous(+/-) mice were mated to generate NBS1 homozygous(-/-) embryos and blastocysts. F1 and F2 mice were maintained and observed for tumor progression.

\section{Embryonic lethality of NBS1-null homozygous(-/-) mice}

Upon intercrossing NBS1 heterozygous mice $(+/-x+/-)$, we were unable to obtain any NBS1 homozygous(-/-) mice from the resulting 108 progeny (Table1). Genotype analysis of these viable mice revealed that 39 mice were wild-type $(+/+)$ and 69 mice were heterozygous(+/-), a ratio of 1:1.8. This strongly suggests that the NBS1 homozygous(-/-) genotype is embryonic lethal, and that NBS1 is essential for embryonic 
Kurimasa et al.

development. To determine the embryonic stage at which normal development of the NBS1-null embryo was arrested, e8.5 to e13.5 embryos were isolated and analyzed by time-mated heterozygous(+/-) intercrossing (Fig.2). Compared to e13.5 heterozygous(+/) and wild-type $(+/+)$ embryos, we observed extremely small resorbed homozygous(-/-) embryos within tiny extra-embryonic tissues (placenta, decidua, yolk sac). These homozygous(-/-) embryos were non-structural and consisted of an organized mass of resorbed tissues. At earlier embryonic stages, e8.5 to e10.5, the size differences between the heterozygous(+/-) and homozygous(-/-) embryos were not so remarkable, and we observed fine structures within homozygous(-/-) embryos. However, homozygous(-/-) embryos were still small compared to heterozygous(+/-) or wild-type $(+/+)$ embryos.

To further confirm the abnormal embryonic development of homozygous(-/-) embryos, we performed histological analysis of e6.5 embryos by sectioning whole deciduas (Fig.3). The size of abnormal embryos was slightly smaller compared to normal embryos, and the number of cells in the abnormal embryos was reduced and tubular structures were disrupted. This suggests that resorbtion of abnormal embryos starts just before the gastrulation period.

The genotypes of embryos obtained from heterozygous(+/-) intercrosses were identified by nested PCR using the core tissues derived from resorbed embryos and tail tissues of embryos with normal appearance (Fig.4, Table 1). PCR results using DNA from normal embryos showed wild-type or heterozygous genotypes. On the other hand, resorbed embryos exhibited homozygous(-/-) genotype.

When we obtained e3.5 blastocysts from heterozygous intercrosses, no obvious structural abnormalities were observed. Genotyping analysis of a total of 135 blastocysts 
Kurimasa et al.

revealed a genetic ratio similar to that expected to arise from Mendelian inheritance, suggesting that NBS1 deficiency does not affect blastocyst development (Table 1). Taken together, these results reveal that development of NBS1 homozygous (-/-) embryos is arrested at an embryonic stage as early as e4.5 to e5.5.

To further analyze the functional importance of NBS1 gene in mouse cells, we attempted to establish cell lines from NBS1 homozygous(-/-) embryonic tissues. Although we sometimes observed a few attached embryonic fibroblast cells around e7.5 and e8.5 embryo tissues, these cells failed to grow and disappeared within a few days. We also attempted to culture the blastocyst cells on feeder cells to establish NBS1 null ES cells (Fig.5). The wild-type $(+/+)$ and heterozygous(+/-) cells from blastocyst inner cell mass (ICM) kept growing under these in vitro culture conditions and were established as ES cells after several passages. On the other hand, cells derived from NBS1 homozygous(-/-) blastocyst ICM stopped growing after 5 days in vitro, and then disappeared. After 7 days in culture, only giant trophoblast cells remained, and replaced the feeder cells.

To verify the manner by which cell death occurred in cells derived from NBS1 homozygous blastocysts within 7 days, blastocyst cells cultured in vitro were analyzed by TUNEL assay to detect apoptosis (Fig.6). The e3.5 blastocysts isolated after heterozygous intercrossing were cultured in DMEM medium. Blastocysts were fixed on the 24 th and 72 nd hour of culture. At the 24 th hour, most of the blastocysts had not yet hatched from the zona pelucida, and contained only a few TUNEL positive cells within the ICM from homozygous(-/-), heterozygous(+/-) and wild-type $(+/+)$ blastocysts. After 3 days in culture, the blastocysts were hatched from the zona pelucida and three- 
dimentional expansion of ICM cells was observed. A large number of TUNEL positive cells within ICM-derived cells were observed in homozygous blastocysts. On the other hand, TUNEL positive cells were still low in number for wild-type $(+/+)$ and heterozygous(+/-) cells. This suggests that NBS1 is essential for early embryonic development and in vitro growth of cells derived from blastocysts, and that NBS1 deficiency causes extensive apoptotic cell death in growing ICM cells.

\section{Tumor progression in NBS1 heterozygous (+/-) mice}

It has been observed that ATM and ATR (ATM/Rad3-related kinase) heterozygous mice have a high incidence of tumor formation $(1,3,4)$. In case of humans, it has also been reported that NBS heterozygous individuals showed an increased incidence of tumor formation(28). To verify the phenotypic effect of heterozygosity $(+/-)$ with respect to tumor incidence and progression, we first measured the ability of ear fibroblasts derived from NBS1 heterozygous(+/-) mice to undergo spontaneous transformation to anchorage-independent growth (Fig.7). Cells derived from wildtype $(+/+)$ or heterozygous $(+/-)$ mice were plated in soft agar and their ability to form colonies was measured after 4 weeks in culture. Most of the cells $(90 \%)$ derived from wild-type $(+/+)$ mice could not form colonies, stopped growing, and remained as single cells. There was no colony observed whose diameter was over $50 \mu \mathrm{m}$. On the other hand, a significant percentage of cells derived from heterozygous mice predominantly formed colonies (54\%) as opposed to remaining as single cells (46\%). Furthermore, $15 \%$ of cells formed colonies larger than $50 \mu \mathrm{m}$ in diameter. NBS1 heterozygous(+/-) cells 
exhibited anchorage-independent growth in soft agar, suggesting that NBS1 heterozygous(+/-) cells readily undergo transformation as compared to wild type cells.

To determine the influence of NBS1 heterozygosity $(+/-)$ on tumor formation in vivo, we examined tumor incidence in heterozygous $(+/-)$ or wild-type $(+/+)$ mice that were over one year-old (Fig.8; Table2). F1 and F2 sibling mice maintained in the same cages were selected at a certain point after they were a year old, euthanized, and autopsied. Internal organs were inspected, and, once pathological abnormalities were observed, abnormal tissues were further examined by histopathology. Finally, a pathological diagnosis was made. Three types of tumors: lung adenocarcinoma $(p=0.41)$ and lymphoma $(\mathrm{p}=0.59)$ from thymus, spleen or lymphnodes were commonly observed in both wild-type $(+/+)$ and heterozygous(+/-) NBS1 mice. On the other hand hepatocellular carcinoma $(\mathrm{HCC})(\mathrm{p}=0.10)$ in liver was observed to occur frequently only in heterozygous mice ( $\mathrm{p}$ value was obtained by Fisher's exact test). All of HCC were observed in male mice.

We further analyzed the loss of heterozygosity of tumors obtained from NBS1 heterozygous( $(+/-)$ mice. Genomic DNA was extracted from tumors and normal liver tissues. Southern hybridization was performed using probes detecting the targeted locus around exon 1. We were unable to detect any loss of heterozygosity (LOH) at the targeted region (data not shown). This strongly suggests that NBS1 gene dosage has an effect on the incidence of specific tumors such as HCC. However, unlike known tumor suppressor genes, a total loss of NBS1 gene product is not sufficient to induce tumor formation since NBS1 homozygous(-/-) cells die just after a few rounds of cell division. 
Kurimasa et al.

\section{DISCUSSION}

We carried out targeted disruption of the NBS1 gene in mice at the transcription start site resulting in null expression of the gene. We observed that development of homozygous(-/-) NBS1 knockout embryos was arrested at a very early stage around e6.5 and that their blastocyst cells were not capable of surviving in vitro culture conditions. Apoptosis was observed in cultured blastocyst cells by TUNEL assay. NBS1 heterozygous $(+/-)$ mice were normal and exhibited no specific phenotype for at least one year. However, fibroblast cells from NBS1 heterozygous(+/-) mice showed enhanced transformation compared to NBS1 wild-type $(+/+)$ cells. After one year, heterozygous (+/-) mice started developing hepatocellular carcinomas (HCC).

There is a clear phenotypic difference between human NBS patients and our knockout homozygous(-/-) mouse model. Even though the onset of human NBS is thought to be at a pre- or early postnatal stage, about $70 \%$ of patients do not show any obvious growth retardation at birth and only exhibit microcephaly in $75 \%$ of cases and sometimes survive for over two decades(32). On the other hand, the homozygous(-/-) null genotype in mice not only results in early embryonic lethality, but most likely lethality even at the cellular level because, in spite of intensive efforts, we were unable to establish homozygous(-/-) cells from blastocysts and early embryos. This correlates very well with previous findings indicating that null mutations in Mre11 and Rad50, which form a functional complex with NBS1, are lethal in mice $(20,38)$. Also, another NBS1 gene knockout constructed by deleting the promoter, exon 1 , and $700 \mathrm{bp}$ of intron 1 showed a similar lethal phenotype(41). Recently, it was reported that the NBS1 gene mutation observed in $90 \%$ of NBS patients (657de15) causes a hypomorphic defect as an 
alternative mode of translation permits expression of a smaller $\mathrm{p} 70$ protein with partial functions(22). The hypomorphic defect has been proposed to diminish the severity of the human NBS phenotype and could explain the lack of lethality in humans that is in contrast to the lethality observed in NBS knockout mice. Also, it was reported very recently that disruption of exons 2 and 3 of the mouse NBS1 gene results in viable mice(14). This discrepancy with our results is probably because in this case the targeted allele can still express an N-terminal truncated NBS1 that, like in humans, could prevent embryonic lethality.

It has been reported that AT carriers have a high risk of cancer, especially breast cancer in women(30). NBS carriers have been shown to have a higher risk of cancerpredisposition than AT carriers, even though the specific cancers were not identified(28). In our study, we observed a high degree of transformation of fibroblast cells derived from NBS1 heterozygous(+/-) mice. In vivo, we also observed a high incidence of a specific tumor, hepatocellular carcinoma $(\mathrm{HCC})$ in heterozygous $(+/-)$ mice. Two other cancers, lung adenocarcinoma and lymphoma, were also detected, but with a nearly equal incidence between wild-type $(+/+)$ and heterozygous $(+/-)$ sibling mice. To understand the mechanism of carcinogenesis observed in NBS1 heterozygous(+/-) mice, we analyzed loss of heterozygosity (LOH) in tumors using a DNA probe complimentary to the targeted locus of the exonl region. We were unable to observe any $\mathrm{LOH}$, which, taken together with the potential cellular lethality of NBS1 homozygous null cells, leads us to speculate that reduced NBS1 gene expression in heterozygous liver cells is sufficient to induce chromosome instability and accelerate cancer progression. 
In the human NBS1 carrier population, there are no obvious tendencies towards liver tumor formation in their cancer spectrum(28). The leading cause of HCC in humans is virus infection by agents such as hepatitis B and C virus, while genetic factors that enhance HCC progression are still unclear(7). In mice, no clear correlation between virus infection and HCC has been identified. On the other hand, incidence of HCC in male mice is very high, suggesting a hormonal effect on HCC occurrence. Our cases of HCC were also observed only in male mice. NBS1 gene expression in liver is very high compared to other tissues as evaluated by northern blot analysis(36). This suggests that in mice liver cells, NBS1 gene expression may be important for maintaining chromosomal integrity and preventing specific cancer (HCC) progression.

The main function of NBS1 protein is still unclear, and it is speculated that NBS1 is involved in both homologous recombination (HR) and non-homologous end-joining (NHEJ) pathways of DNA double-strand break repair(15, 33). Knockouts of several genes related to the NHEJ pathway do not show any lethality (Ku70, Ku80 or DNA$\mathrm{PKcs})(16,24,25)$ or show embryonic lethality only at a late stage(XRCC4, LigaseIV)(10, 11). Furthermore, null of expression of ATM, which is involved in DNA damage recognition and signaling to the cell cycle machinery, does not lead to lethality $(2,39)$. On the other hand, mice with deletions of HR-related genes show early embryonic lethality $(8,17,19,29,31)$, suggesting that HR is essential for early mouse development. From the embryonic lethality of NBS1 knockout mice and the lethality of knockouts of its two interacting proteins, Mre11 and Rad50, the main function of NBS1 or Mre11/Rad50/NBS1 complex would appear to be related to the HR pathway. 
Kurimasa et al.

With the failure to establish a mouse model for human NBS and to obtain viable NBS1 deficient cell lines, we are currently constructing a conditional knockout for the NBS1 gene and knockin mice carrying the common mutation (657del5) observed in human NBS patients. By further studying these mice in detail, it will become clear as to how loss of the NBS1 gene may contribute to cancer progression and why NBS1 is essential for early mouse development. 
Kurimasa et al.

\section{ACKNOWLEDGEMENT}

We thank Ms. Candice GC Tahimic for critical reading of manuscript, and Ms. Paige Pardington, Dr. Li-jin Dong, and Dr. Xi-Ling Wu for technical assistance. This work was funded by the US Dept. of Energy and NIH grants AG17709 and CA50519 to D.J.C. 


\section{FIGURE LEGEND}

Figure 1 (A) Schematic diagram of mouse NBS1 gene locus (exon1 to 5) and Southern hybridization probe A (Top), the targeting construct (Middle) and the targeted allele (Bottom). E: EcoRI, P: PstI. (B) PCR analysis of targeted ES cells. Using primers indicated on the map, a $1.3 \mathrm{~kb}$ DNA fragment was amplified only from homologously-targeted ES cells (Clone \#2,6,15,18 and 22). M: marker, P: positive control, N: negative control, B: Blank. (C) Southern blot analysis of EcoRI-digested DNA from targeted ES cells. Targeted ES cells (Clone \#2, 6, 15, 18 and 22) show two signals, one from the wild type allele $(11 \mathrm{~kb})$ and the other from the targeted allele $(5 \mathrm{~kb})$.

Figure 2 Normal NBS1 heterozygous(+/-) and resorbed NBS1 homozygous(-/-) embryos. Heterozygous(+/-) e13.5 embryo is shown with extraembryonic tissues (placenta, yolk sac)(A), and embryo only(B). Homozygous(-/-) e13.5 embryo with extraembryonic tissue $(\mathbf{C})$ is smaller than heterozygous(+/-) embryo. Comparison of a heterozygous (+/-) and two homozygous(-/-) e10.5 embryos with deciduas are shown in (D). Embryos isolated from (D) are shown in (E) $(+/-),($ F) $(-/-)$, and $($ G)(-/-). Comparison of heterozygous(+/-) and homozygous(-/-) e8.5 embryo with decidua(H). Sectioned view of homozygous embryo with decidua from (H, right) are shown in (I). Embryo undergoing resorbtion is centrally-located in the top part of the section(I). Comparison of heterozygous(+/-) and homozygous(-/-) e8.5 embryos (J).

Figure 3 Histological analysis of e6.5 embryos obtained from heterozygous intercrossing. Hematoxylin and eosin staining of control normal embryos(A, B) and 
abnormal embryos $(\mathbf{C}, \mathbf{D})$. The abnormal embryo had not developed appreciably beyond e5.5-e6.0 stage and had started deteriorating. eme: embryonic ectoderm, pc: proamniotic cavity, exe: extraembryonic ectoderm.

Figure 4 Example of PCR genotyping of cultured blastocysts subsequently TUNEL stained as described in Table1, Fig.5 and Fig.6 are shown in (A). PCR genotyping using embryonic tissue (Table1) is shown in (B). Specifically amplified PCR products from wild type or knockout alleles were transferred to nitrocellulose membrane and hybridized with ${ }^{32} \mathrm{P}$ end-labeled internal primers specific for wild-type or knockout PCR products.

Figure 5 NBS1 homozygous(-/-) cells from blastocyst (e3.5) are unable to proliferate in vitro. The e 3.5 blastocysts were isolated from NBS1 heterozygous(+/-) intercrosses and were cultured with feeder cells (MEFs). After 2 days of culture, NBS1 wild-type $(+/+)$, heterozygous(+/-) and homozygous(-/-) blastocysts hatched from the zona pellucida and implanted onto feeder cells. During the first 3 days of culture, the inner cell mass(ICM) of NBS1 homozygous(-/-) blastocysts were indistinguishable from that of wild-type $(+/+)$ or heterozygous(+/-) blastocysts. However, while the ICM cells of wild-type(+/+) and heterozygous(+/-) blastocysts continued to expand, NBS1 homozygous(-/-) ICM cells stopped expanding after 5 days.

Figure 6 Disruption of NBS1 leads to apoptotic cell death of early embryonic cells. TUNEL staining of cultured blastocysts obtained from NBS1 heterozygous(+/-) 
Kurimasa et al.

intercrosses were performed after $24 \mathrm{hr}$ (A), and $72 \mathrm{hrs}$ (B) of culture. Phase contrast, Fluorescin (TUNEL, green), and DAPI(blue) or PI(red) images are shown. Many TUNEL positive cells were observed in NBS1 homozygous(-/-) blastocysts especially after $72 \mathrm{hrs}$ of culture.

\section{Figure 7 Enhanced Transformation of NBS1 Heterozygous(+/-) Primary} Fibroblast Cells. NBS1 wild-type $(+/+)$ fibroblast cells were unable to form colonies, and remained as single cells in soft agar(A). On the other hand, NBS1 heterozygous $(+/-)$ fibroblast cells formed colonies of various sizes, some over $50 \mu \mathrm{m}$ in $\operatorname{diameter}(\mathbf{B}, \mathbf{C}, \mathbf{D})$. Bar graph(E) represents the size distribution of colonies in soft agar from wild-type $(+/+)$ and NBS1 heterozygous(+/-) primary fibroblast cells.

\section{Figure 8 Histopathological analysis of tumors obtained from NBS} heterozygous(+/-) mice. Three different type of tumors were observed: lung adenocarcinoma (A x5, B x200), lymphoma from peri-pancreous lymphnode (C x5, D x200) and hepatocellular carcinoma(HCC) $(\mathbf{E} \times 20, \mathbf{F} \times 200)$. HCC were obtained only from heterozygous $(+/-)$ male mice, but not from wild-type $(+/+)$ mice. Bar graph represents tumor incidence among wild-type $(+/+)$ and NBS1 heterozygous $(+/-)$ mice observed after 1 year. The p values were calculated by Fisher's exact test. 
Kurimasa et al.

\section{REFERENCE}

1. Barlow, C., M. A. Eckhaus, A. A. Schaffer, and A. Wynshaw-Boris. 1999. Atm haploinsufficiency results in increased sensitivity to sublethal doses of ionizing radiation in mice. Nat Genet 21:359-60.

2. Barlow, C., S. Hirotsune, R. Paylor, M. Liyanage, M. Eckhaus, F. Collins, Y. Shiloh, J. N. Crawley, T. Ried, D. Tagle, and A. Wynshaw-Boris. 1996. Atmdeficient mice: a paradigm of ataxia telangiectasia. Cell 86:159-71.

3. Bay, J. O., N. Uhrhammer, D. Pernin, N. Presneau, A. Tchirkov, M. Vuillaume, V. Laplace, M. Grancho, P. Verrelle, J. Hall, and Y. J. Bignon. 1999. High incidence of cancer in a family segregating a mutation of the ATM gene: possible role of ATM heterozygosity in cancer. Hum Mutat 14:485-92.

4. Brown, E. J., and D. Baltimore. 2000. ATR disruption leads to chromosomal fragmentation and early embryonic lethality. Genes Dev 14:397-402.

5. Carney, J. P. 1999. Chromosomal breakage syndromes. Curr Opin Immunol 11:443-7.

6. Carney, J. P., R. S. Maser, H. Olivares, E. M. Davis, M. Le Beau, J. R. Yates, 3rd, L. Hays, W. F. Morgan, and J. H. Petrini. 1998. The hMre11/hRad50 protein complex and Nijmegen breakage syndrome: linkage of double-strand break repair to the cellular DNA damage response. Cell 93:477-86.

7. de La Coste, A., B. Romagnolo, P. Billuart, C. A. Renard, M. A. Buendia, O. Soubrane, M. Fabre, J. Chelly, C. Beldjord, A. Kahn, and C. Perret. 1998. Somatic mutations of the beta-catenin gene are frequent in mouse and human hepatocellular carcinomas. Proc Natl Acad Sci U S A 95:8847-51.

8. Deans, B., C. S. Griffin, M. Maconochie, and J. Thacker. 2000. Xrcc2 is required for genetic stability, embryonic neurogenesis and viability in mice. Embo J 19:6675-85.

9. Digweed, M., A. Reis, and K. Sperling. 1999. Nijmegen breakage syndrome: consequences of defective DNA double strand break repair. Bioessays 21:649-56.

10. Frank, K. M., J. M. Sekiguchi, K. J. Seidl, W. Swat, G. A. Rathbun, H. L. Cheng, L. Davidson, L. Kangaloo, and F. W. Alt. 1998. Late embryonic lethality and impaired $\mathrm{V}(\mathrm{D}) \mathrm{J}$ recombination in mice lacking DNA ligase IV. Nature 396:173-7.

11. Gao, Y., Y. Sun, K. M. Frank, P. Dikkes, Y. Fujiwara, K. J. Seidl, J. M. Sekiguchi, G. A. Rathbun, W. Swat, J. Wang, R. T. Bronson, B. A. Malynn, M. Bryans, C. Zhu, J. Chaudhuri, L. Davidson, R. Ferrini, T. Stamato, S. H. Orkin, M. E. Greenberg, and F. W. Alt. 1998. A critical role for DNA endjoining proteins in both lymphogenesis and neurogenesis. Cell 95:891-902.

12. Gatei, M., D. Young, K. M. Cerosaletti, A. Desai-Mehta, K. Spring, S. Kozlov, M. F. Lavin, R. A. Gatti, P. Concannon, and K. Khanna. 2000. ATMdependent phosphorylation of nibrin in response to radiation exposure. Nat Genet 25:115-9.

13. Huo, Y. K., Z. Wang, J. H. Hong, L. Chessa, W. H. McBride, S. L. Perlman, and R. A. Gatti. 1994. Radiosensitivity of ataxia-telangiectasia, X-linked agammaglobulinemia, and related syndromes using a modified colony survival assay. Cancer Res 54:2544-7.

14. Kang, J., R. T. Bronson, and Y. Xu. 2002. Targeted disruption of NBS1 reveals its roles in mouse development and DNA repair. Embo J 21:1447-55. 
15. Khanna, K. K., and S. P. Jackson. 2001. DNA double-strand breaks: signaling, repair and the cancer connection. Nat Genet 27:247-54.

16. Kurimasa, A., H. Ouyang, L. J. Dong, S. Wang, X. Li, C. Cordon-Cardo, D. J. Chen, and G. C. Li. 1999. Catalytic subunit of DNA-dependent protein kinase: impact on lymphocyte development and tumorigenesis. Proc Natl Acad Sci U S A 96:1403-8.

17. Lim, D. S., and P. Hasty. 1996. A mutation in mouse rad51 results in an early embryonic lethal that is suppressed by a mutation in p53. Mol Cell Biol 16:713343.

18. Lim, D. S., S. T. Kim, B. Xu, R. S. Maser, J. Lin, J. H. Petrini, and M. B. Kastan. 2000. ATM phosphorylates p95/nbs1 in an S-phase checkpoint pathway. Nature 404:613-7.

19. Liu, C. Y., A. Flesken-Nikitin, S. Li, Y. Zeng, and W. H. Lee. 1996. Inactivation of the mouse Brcal gene leads to failure in the morphogenesis of the egg cylinder in early postimplantation development. Genes Dev 10:1835-43.

20. Luo, G., M. S. Yao, C. F. Bender, M. Mills, A. R. Bladl, A. Bradley, and J. H. Petrini. 1999. Disruption of mRad50 causes embryonic stem cell lethality, abnormal embryonic development, and sensitivity to ionizing radiation. Proc Natl Acad Sci U S A 96:7376-81.

21. MacPherson, I. 1973. Soft agar techniques, p. 276-280. In P. Kruse Jr., and M. Patterson Jr. (ed.), Tissue Culture Methods and applications. Academic Press, New York.

22. Maser, R. S., R. Zinkel, and J. H. Petrini. 2001. An alternative mode of translation permits production of a variant NBS1 protein from the common Nijmegen breakage syndrome allele. Nat Genet 27:417-21.

23. Matsuura, S., H. Tauchi, A. Nakamura, N. Kondo, S. Sakamoto, S. Endo, D. Smeets, B. Solder, B. H. Belohradsky, V. M. Der Kaloustian, M. Oshimura, M. Isomura, Y. Nakamura, and K. Komatsu. 1998. Positional cloning of the gene for Nijmegen breakage syndrome. Nat Genet 19:179-81.

24. Nussenzweig, A., C. Chen, V. da Costa Soares, M. Sanchez, K. Sokol, M. C. Nussenzweig, and G. C. Li. 1996. Requirement for Ku80 in growth and immunoglobulin V(D)J recombination. Nature 382:551-5.

25. Ouyang, H., A. Nussenzweig, A. Kurimasa, V. C. Soares, X. Li, C. CordonCardo, W. Li, N. Cheong, M. Nussenzweig, G. Iliakis, D. J. Chen, and G. C. Li. $1997 . \mathrm{Ku} 70$ is required for DNA repair but not for T cell antigen receptor gene recombination In vivo. J Exp Med 186:921-9.

26. Petrini, J. H. 1999. The mammalian Mre11-Rad50-nbs1 protein complex: integration of functions in the cellular DNA-damage response. Am J Hum Genet 64:1264-9.

27. Petrini, J. H. 2000. The Mre11 complex and ATM: collaborating to navigate S phase. Curr Opin Cell Biol 12:293-6.

28. Seemanova, E. 1990. An increased risk for malignant neoplasms in heterozygotes for a syndrome of microcephaly, normal intelligence, growth retardation, remarkable facies, immunodeficiency and chromosomal instability. Mutat Res 238:321-4.

29. Sharan, S. K., M. Morimatsu, U. Albrecht, D. S. Lim, E. Regel, C. Dinh, A. Sands, G. Eichele, P. Hasty, and A. Bradley. 1997. Embryonic lethality and radiation hypersensitivity mediated by $\operatorname{Rad} 51$ in mice lacking Brca2. Nature 386:804-10. 
30. Swift, M., D. Morrell, R. B. Massey, and C. L. Chase. 1991. Incidence of cancer in 161 families affected by ataxia-telangiectasia. $N$ Engl J Med 325:18316.

31. Tsuzuki, T., Y. Fujii, K. Sakumi, Y. Tominaga, K. Nakao, M. Sekiguchi, A. Matsushiro, Y. Yoshimura, and MoritaT. 1996. Targeted disruption of the Rad51 gene leads to lethality in embryonic mice. Proc Natl Acad Sci U S A 93:6236-40.

32. van der Burgt, I., K. H. Chrzanowska, D. Smeets, and C. Weemaes. 1996. Nijmegen breakage syndrome. J Med Genet 33:153-6.

33. van Gent, D. C., J. H. Hoeijmakers, and R. Kanaar. 2001. Chromosomal stability and the DNA double-stranded break connection. Nat Rev Genet 2:196206.

34. Varon, R., C. Vissinga, M. Platzer, K. M. Cerosaletti, K. H. Chrzanowska, K. Saar, G. Beckmann, E. Seemanova, P. R. Cooper, N. J. Nowak, M. Stumm, C. M. Weemaes, R. A. Gatti, R. K. Wilson, M. Digweed, A. Rosenthal, K. Sperling, P. Concannon, and A. Reis. 1998. Nibrin, a novel DNA double-strand break repair protein, is mutated in Nijmegen breakage syndrome. Cell 93:467-76.

35. Wang, Y., D. Cortez, P. Yazdi, N. Neff, S. J. Elledge, and J. Qin. 2000. BASC, a super complex of BRCAl-associated proteins involved in the recognition and repair of aberrant DNA structures. Genes Dev 14:927-39.

36. Wilda, M., I. Demuth, P. Concannon, K. Sperling, and H. Hameister. 2000. Expression pattern of the Nijmegen breakage syndrome gene, Nbs1, during murine development. Hum Mol Genet 9:1739-44.

37. Wu, X., V. Ranganathan, D. S. Weisman, W. F. Heine, D. N. Ciccone, T. B. O'Neill, K. E. Crick, K. A. Pierce, W. S. Lane, G. Rathbun, D. M. Livingston, and D. T. Weaver. 2000. ATM phosphorylation of Nijmegen breakage syndrome protein is required in a DNA damage response. Nature 405:477-82.

38. Xiao, Y., and D. T. Weaver. 1997. Conditional gene targeted deletion by Cre recombinase demonstrates the requirement for the double-strand break repair Mre11 protein in murine embryonic stem cells. Nucleic Acids Res 25:2985-91.

39. Xu, Y., T. Ashley, E. E. Brainerd, R. T. Bronson, M. S. Meyn, and D. Baltimore. 1996. Targeted disruption of ATM leads to growth retardation, chromosomal fragmentation during meiosis, immune defects, and thymic lymphoma. Genes Dev 10:2411-22.

40. Zhao, S., Y. C. Weng, S. S. Yuan, Y. T. Lin, H. C. Hsu, S. C. Lin, E. Gerbino, M. H. Song, M. Z. Zdzienicka, R. A. Gatti, J. W. Shay, Y. Ziv, Y. Shiloh, and E. Y. Lee. 2000. Functional link between ataxia-telangiectasia and Nijmegen breakage syndrome gene products. Nature 405:473-7.

41. Zhu, J., S. Petersen, L. Tessarollo, and A. Nussenzweig. 2001. Targeted disruption of the Nijmegen breakage syndrome gene NBS1 leads to early embryonic lethality in mice. Curr Biol 11:105-9.

42. Zhu, X. D., B. Kuster, M. Mann, J. H. Petrini, and T. Lange. 2000. Cell-cycleregulated association of RAD50/MRE11/NBS1 with TRF2 and human telomeres. Nat Genet 25:347-52. 
Figure 1 Kurimasa et al.

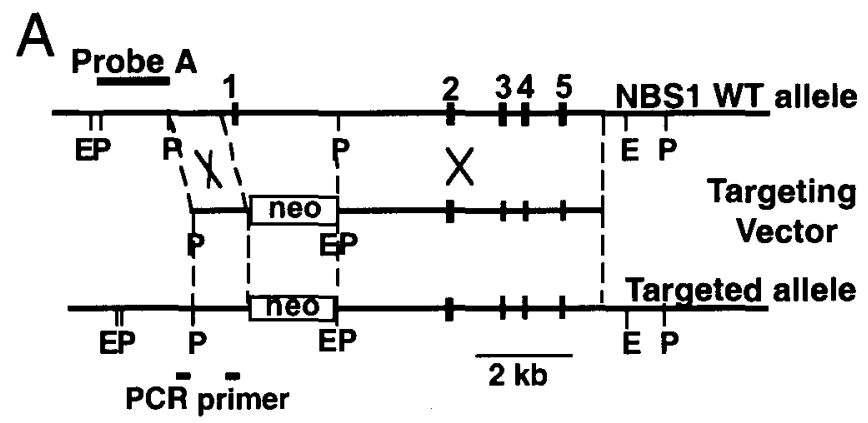

B

ES clone

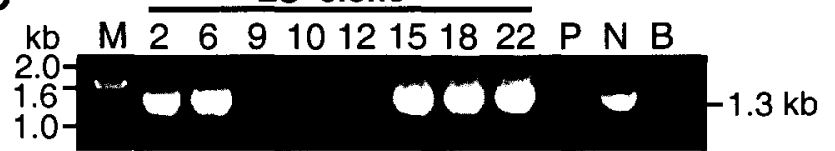

C

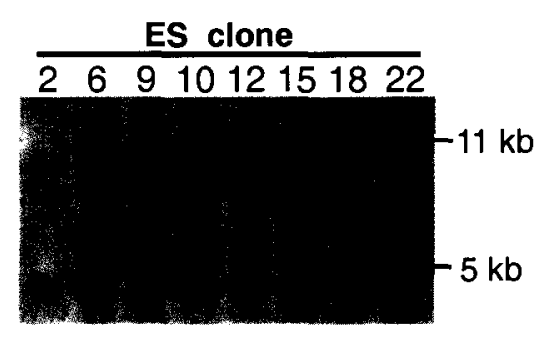


Figure 2 Kurimasa et al.
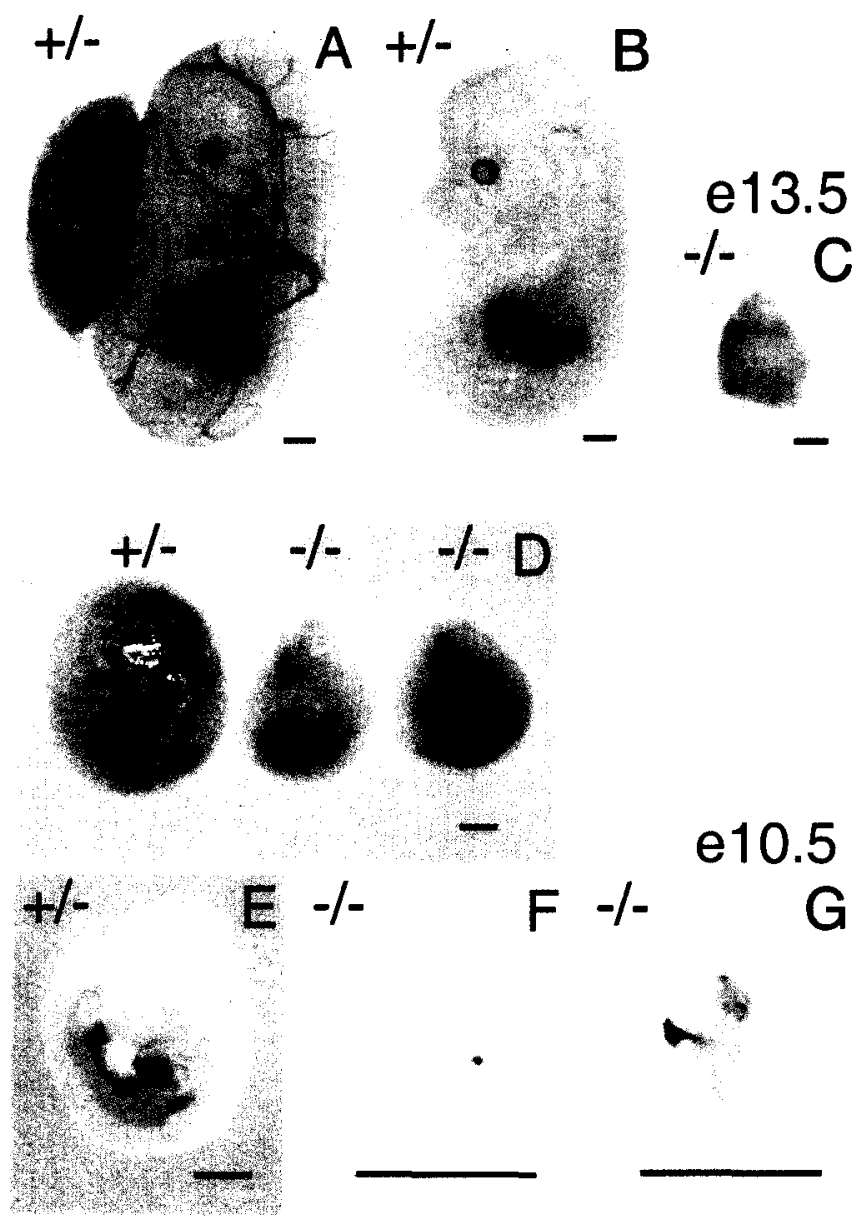

$+/-\quad-1-11 \quad-1-1$
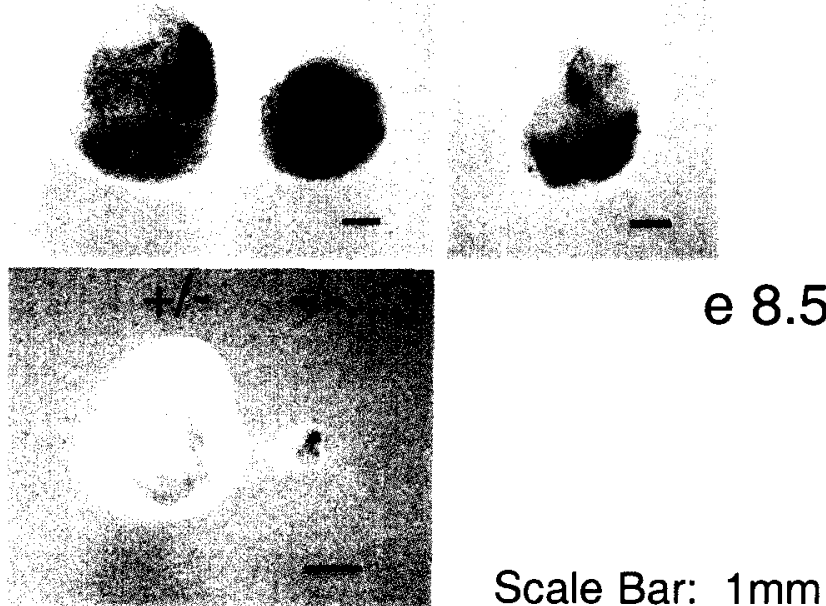

Scale Bar: $1 \mathrm{~mm}$ 
Figure 3 Kurimasa et al.

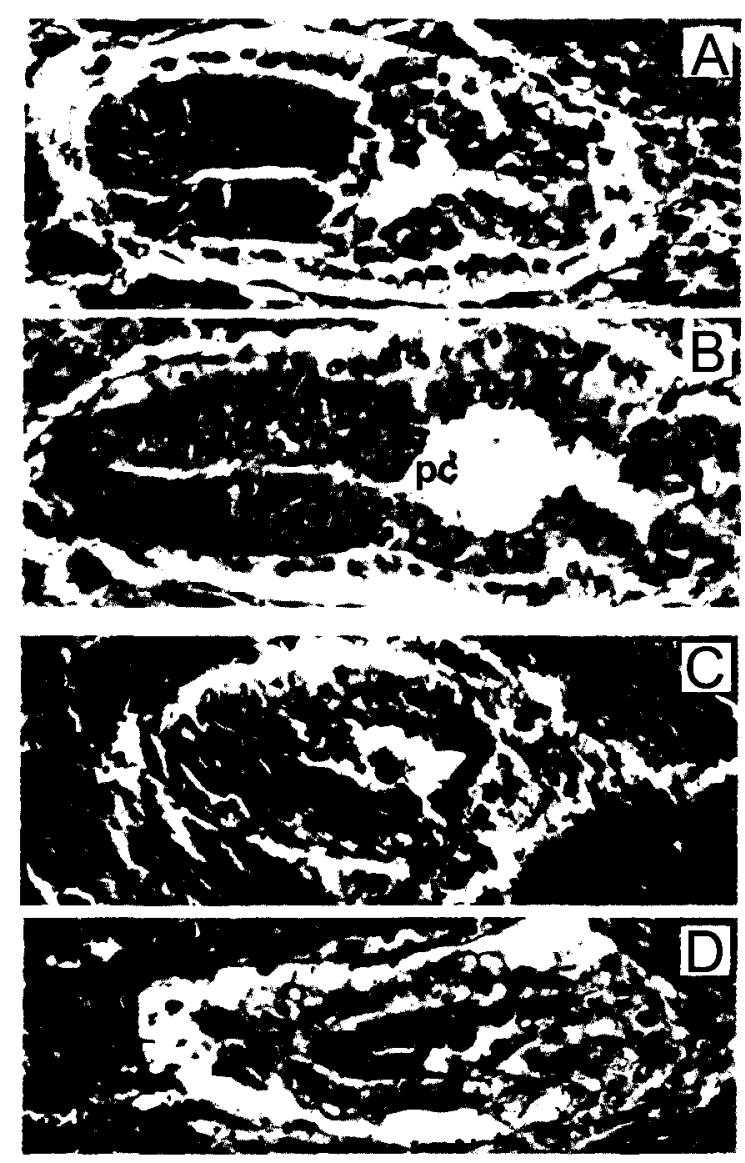


Figure 4 Kurimasa et al.

A Blastocysts

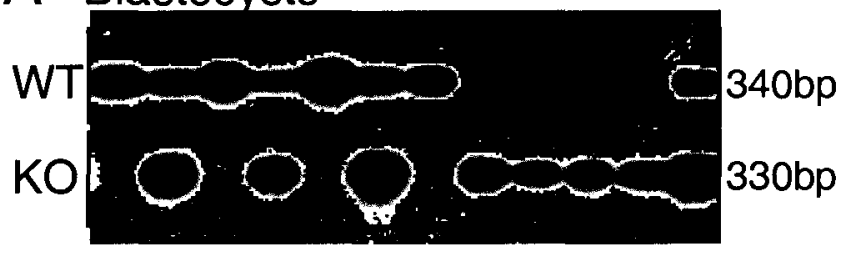

B Embryo Tissue

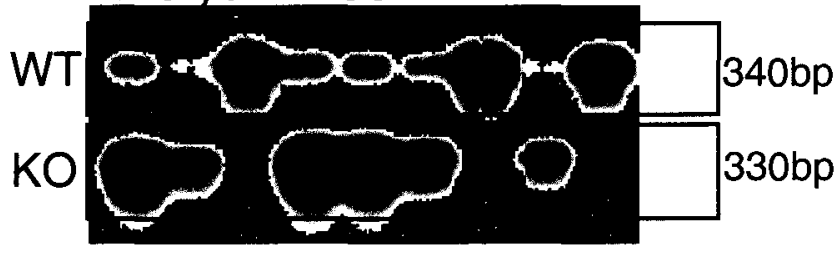




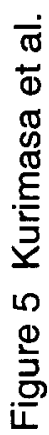

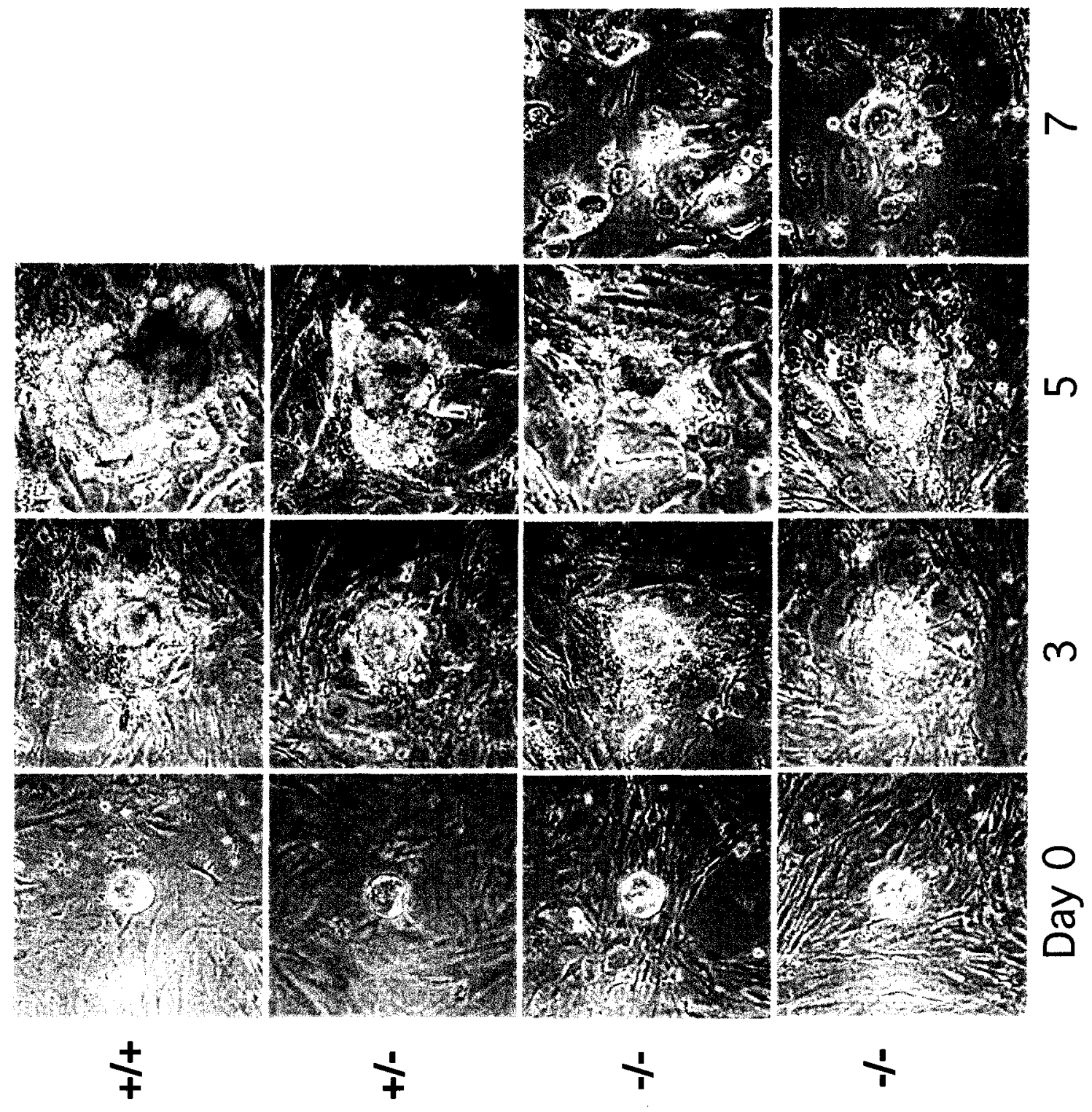




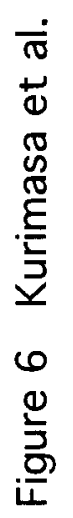
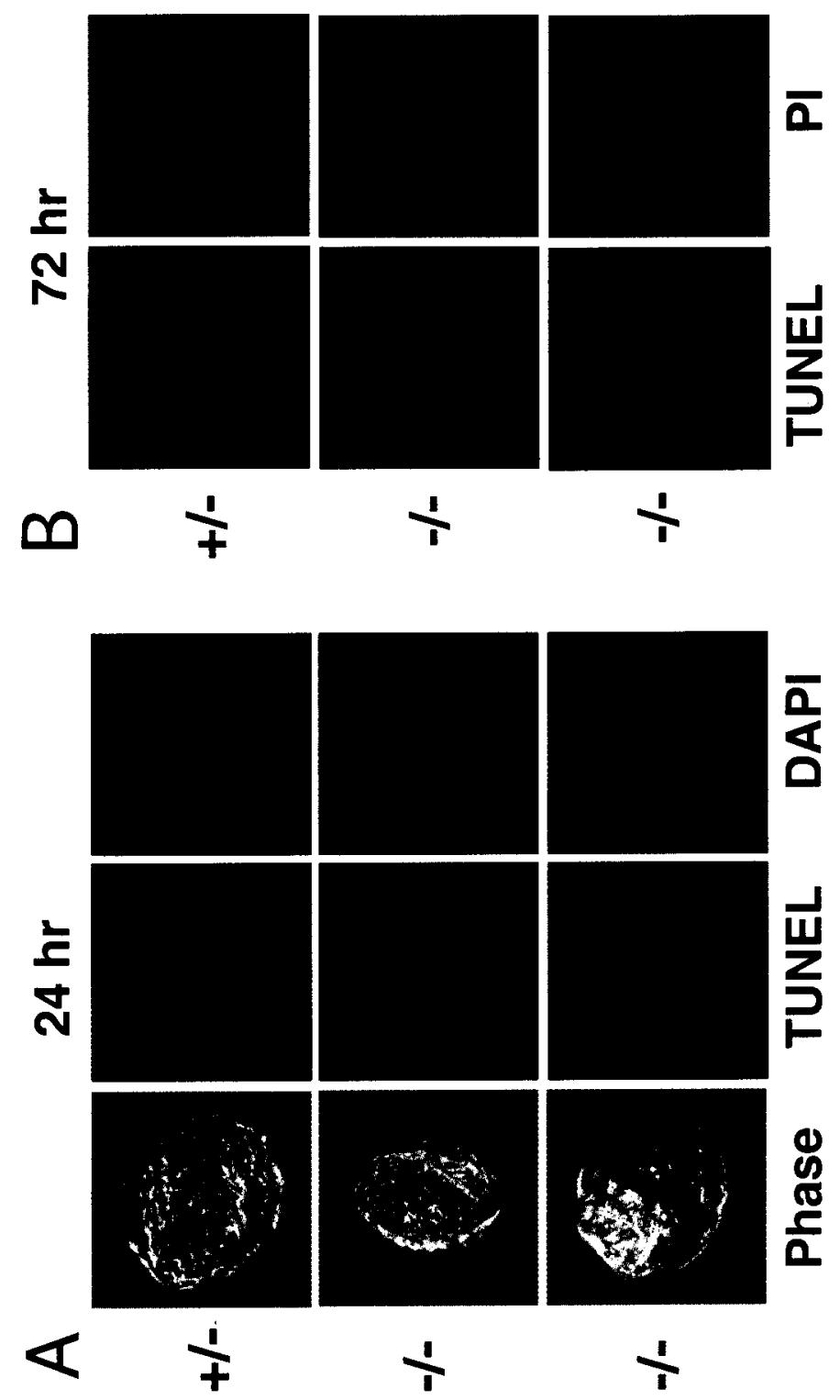


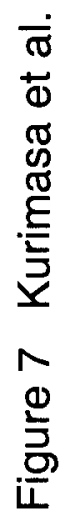

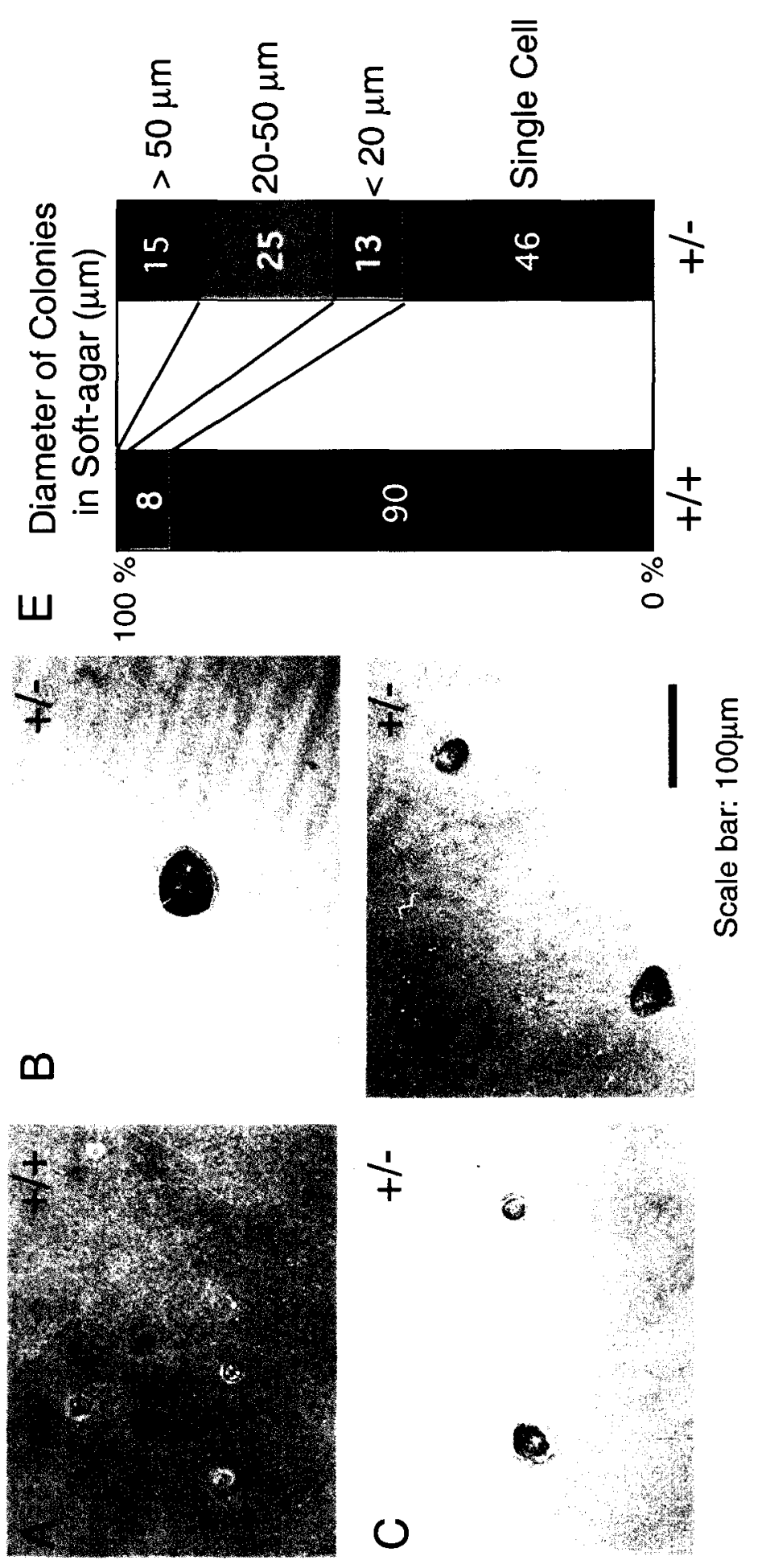




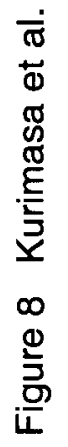
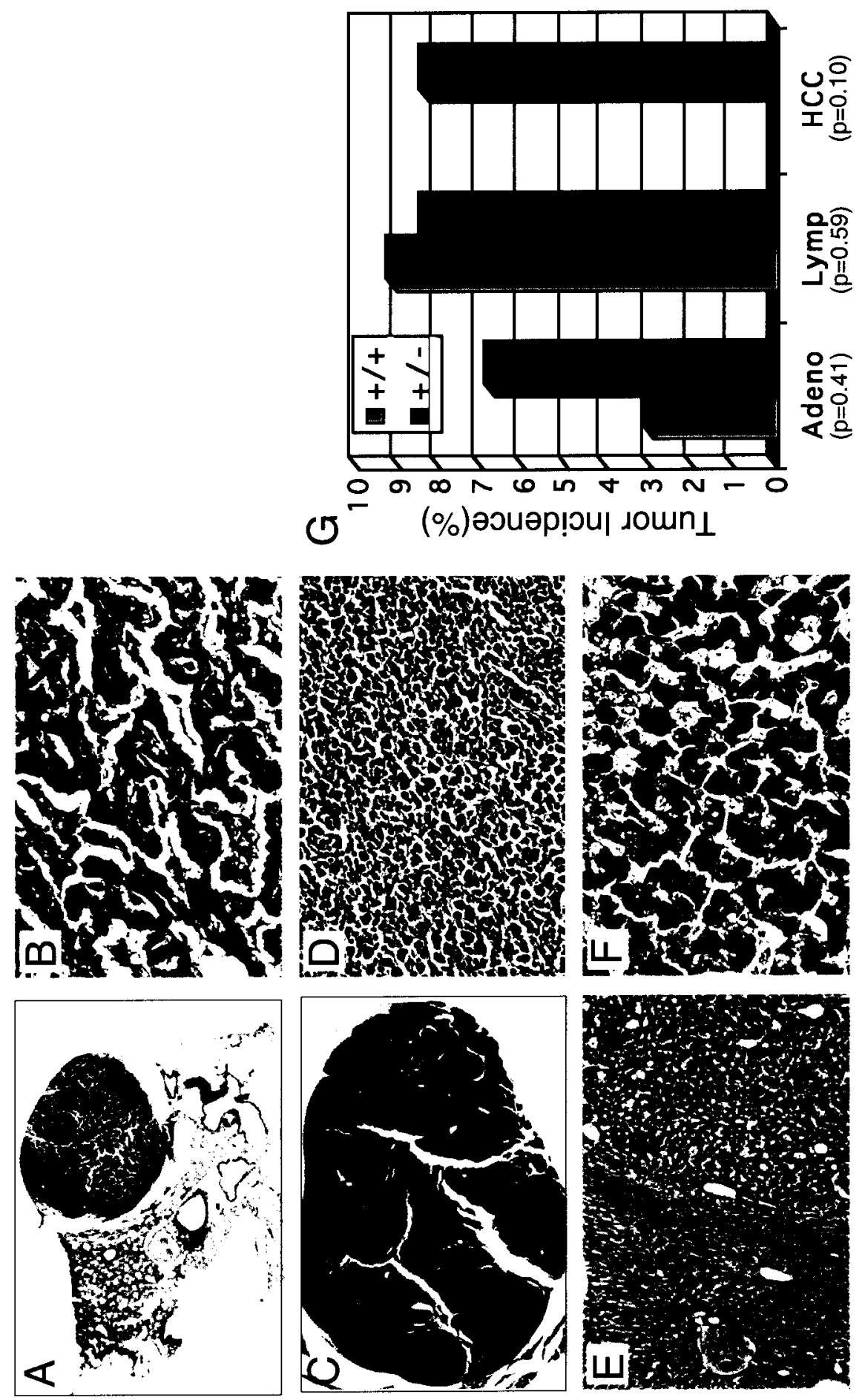